\title{
The Prevalence, Trend, and Associated Demographic Factors of Neural Tube Defects at Orotta National Referral Maternity Hospital, Asmara: Retrospective Record Review Study
}

\author{
Dawit Estifanos $^{1, *}$, Elias Teages Adgoy ${ }^{2, *}$, Dawit Sereke ${ }^{1}$, Birhane Zekarias ${ }^{1}$, Suzan Marzolf $^{1}$, \\ Kifleyesus Tedla ${ }^{1}$ \\ ${ }^{1}$ Department of Obstetrics and Gynaecology, Ministry of Health, Asmara, Eritrea \\ ${ }^{2}$ Department of Community Medicine and Primary Health Care, Orotta School of Medicine and Dentistry, Asmara, Eritrea
}

Email address:

destifanos50@yahoo.com (D. Estifanos), eliasteages@gmail.com (E. T. Adgoy), weldegebrealdawit@gmail.com (D. Sereke), Zekariasberhane@gmail.com (B. Zekarias),smeritrea@gmail (S. Marzolf), pkiflevita@gmail (K. Tedla)

${ }^{*}$ Corresponding author

\section{To cite this article:}

Dawit Estifanos, Elias Teages Adgoy, Dawit Sereke, Birhane Zekarias, Suzan Marzolf, Kifleyesus Tedla. The Prevalence, Trend, and Associated Demographic Factors of Neural Tube Defects at Orotta National Referral Maternity Hospital, Asmara: Retrospective Record Review Study. Science Journal of Public Health. Vol. 5, No. 6, 2017, pp. 452-456. doi: 10.11648/j.sjph.20170506.17

Received: October 5, 2017; Accepted: October 23, 2017; Published: November 17, 2017

\begin{abstract}
Neural Tube Defects (NTDs) are congenital structural abnormalities of the brain (anencephaly) and vertebral column (spina bifida) that represented as one of the most common congenital malformations of neonates worldwide. A fouryear and eight months (January $1^{\text {st }}, 2007$ to August $31^{\text {st }}$, 2011) retrospective record review study on prevalence, trend, and associated demographic factors of NTD was conducted at Orotta National Referral Maternity Hospital, Asmara, Eritrea. The prevalence, trend and associated demographic factors were assessed by data extraction from hospital delivery register and patient cards. Associations between variables of maternal age, parity, and infant gender and primary outcomes were determined using $\chi^{2}$ analyses and Poisson regression modeled cumulative incidence and controlled for confounders. Out of 39,803 total deliveries 185 neonates were found to have NTDs related abnormalities, but 156 neonates identified with complete and welldocumented registers, medical records and charts showing a prevalence of 3.9 per 1000 deliveries. The most commonly identified NTDs were Anencephaly 75(48.1\%), Hydrocephalus 29 (18.6\%), Spina bifida 27 (17.3\%), and Multiple NTDs 25 $(16.0 \%)$. Marital status, ethnicity, religion, and maternal history of abortion were found to have no enough evidence, but women who delivered neonates with NTDs who had parity less than four $125(80.1 \%)$ and age below 30 years 108 (69.2\%)were observed to have higher chances. All the Anencephalic neonates were delivered by vaginal delivery, Majority (66 $(88 \%)$ ) with a weight of less than 2000 grams and were female by sex 55(74.3\%); whereas 13(44.8\%) Hydrocephalic neonates were delivered by Caesarian section and $26(92.9 \%)$ had a weight greater than 2000 grams. The Appearance Pulse Grimace Activity Respiration(APGAR) score results were founded to be zero for all anencephalic neonates and for 17 (74\%) of multiple NTD; while 40 percent of and 46 percent of neonates with spina bifida and hydrocephalus, respectively, had no APGAR at five minutes. Over the years of the study a significant increase in the trend of NTDs were observed, mainly that of anencephaly. The increase was 1.5 percent $(\mathrm{p}<0.05)$. The results of Poisson analysis indicated an exceptional statistically significant increase of anencephaly only. The trend of NTDs in neonates was observed to increase significantly, mainly anencephaly. The study findings noted younger women (less than 30 years) and with parity less than four were at higher risk than those of older age and higher parity. NTDs can be prevented and reduced with folic acid supplementation and fortification of principal foods.
\end{abstract}

Keywords: Neural Tube Defects, Deliveries, Neonate, Congenital Structural Abnormalities 


\section{Introduction}

This Neural Tube Defect (NTD) is represented as one of the most common congenital malformations of neonates worldwide. NTD are congenital structural abnormalities of the brain (anencephaly) and vertebral column (spina bifida) that occur either as an isolated malformation alone, in combination with other malformations, or as part of a genetic syndrome. Isolated (nonsyndromic) NTDs occur in $1.4-2$ per 1, 000 pregnancies and are the second most common major congenital anomaly worldwide. Anencephaly accounts for one half of all cases of NTD and is incompatible with life; 80-90 percent of infants with spina bifida can survive with treatment having varying degrees of disability [1].

The exact cause of NTD is unknown; over 95 percent of NTD occur in couples with a negative family history. Nevertheless, the risk of recurrence is 1 in 33 couples in those who were affected within one pregnancy and 1 in 10 in those who were affected within two pregnancies [2]. Sisters of women with an affected child have a chance of 1 in 100 and sisters of a man with an affected child have a chance of 1 in 300 risks for acquiring NTD. Deficiency related to Folic acid and Zinc was proposed as possible causes of NTDs until now, as it is documented in many studies maternal diabetes, alcohol abuse, and antenatal irradiation are some of the known high risk factors for NTD whereas, anticonvulsant therapy, maternal hyperthermia, antenatal exposure to rubella and hallucinogen ingestion are considered as suspected contributing factors [2].

In early to mid 1990's, multiple clinical studies revealed that preconception ingestion of folic acid decreases the risk of NTD as much as by $50-70$ percent $[4,5]$. Therefore, based on the above studies worldwide active implementation of educational campaigns for preconception folic acid supplementation and food fortification for reproductive age women is practice until now [2]. In late 1990's, many high income developed countries implemented country wide food and prenatal vitamin fortification. The results of follow up studies that conducted in many countries indicated the prevalence and trend of NTD was observed to drop as it was expected after the interventions of fortification. In addition to nutritional studies, other socio demographic characteristics such as age, ethnicity and antenatal use of anticonvulsants were also suggested as determinants for NTD. In Africa, several countries have documented a higher frequency of NTD than before; a study conducted by Djientcheu, et. al (2008) and Adeleye, et. al (2010) indicated an incidence rates of 1.99 per 1000 births in Cameroon and 7 per 1000 births in Nigeria [4, 6]. Until now there are no studies that are published on prevalence, trend and associated demographic factors of NTD in Eritrea. Therefore, the purpose of this study to obtain data on NTD prevalence, trends, and associated demographic factors for Eritrea.

\section{Materials and Methods}

\subsection{Study Participants and Study Period}

All deliveries above twenty-eight weeks of gestation at
Orotta National Referral Maternity Hospital (OMNRH), from January $1^{\text {st }}, 2007$ to August $31^{\text {st }}, 2011$ (four years and eight months) were included. Out of 39, 803 total deliveries there were 185 neonates who had delivered with NTD related abnormalities but only 156 neonates with NTD were included because they had a well-documented registers, medical records and/or charts and additional information that were documented in their patient charts.

\subsection{Data Collection, Processing and Analysis}

Data of all deliveries above twenty-eight weeks of gestation, from January $1^{\text {st }}, 2007$ to August $31^{\text {st }}$, 2011(four years and eight months) were collected from Orotta Maternity National Referral Hospital by looking and comparing at the records of delivery register book and patients charts. Register books containing information on patient name, age, address, gravidity, and parity, mode of delivery, dates and neonatal outcomes of APGAR (Appearance Pulse Grimace Activity Respiration), weight, gender and comments of abnormities were documented. Additional information was also extracted from the patient charts that included marital status, religion, antenatal care (ANC) follow up, ethnicity, maternal medical history, medication history and gestational age.

For data analysis purpose an overall and NTD type specific prevalence were calculated by dividing the total counts of each defect by all deliveries, per 1000 total births. To facilitate the assessment of specific prevalence differences and relative risk across categories of each maternal demographic variable studied, Poisson regression was employed to calculate prevalence ratios (PR) at 95\% confidence interval $(\mathrm{CI})$ with one category serving as the reference for each variable. For data analysis STATA 9.1 was used in order to fit log linear models and calculate parameter estimates. The estimates, lower and upper CI were exponentiated to obtain prevalence with $95 \%$ CIs. We fitted the Poisson regression model with scaling factor option. The significance linear trends for delivery month, year, maternal, age, marital status, address, mode of delivery, parity, gender, birth weight, gestational age, ethnicity, religion, maternal history and medication was also examined using Poisson regression to check for trend and unadjusted analyses using ordinary coded variables. The variables which showed linear trends $(\mathrm{P}<0.05)$ were included in the final multivariable analyses.

\section{Results}

There were a total of 39,803 total deliveries (live births and stillbirths) from January $1^{\text {st }}, 2007$ to August $31^{\text {st }}, 2011$ (within 56 months). Out of these deliveries there were 185 neonates who had delivered with NTD related abnormalities but only 156 neonates with NTD were found to have a welldocumented registers, medical records and/or charts resulting to a prevalence of 3.9 per 1000 total deliveries. The study results revealed that Anencephaly was the most common type 
75(48.1\%) of NTD followed by hydrocephalus 29 (18.6\%), Spina bifida $27(17.3 \%)$, and multiple NTD 25 (16.0\%).

Table 1. Socio-Demographic Characteristics of Mother who delivered NTDs.

\begin{tabular}{|c|c|c|c|c|c|c|}
\hline Maternal Characteristics & & Total NTD & Anencephaly & Spina-bifida & Hydrocephalus & Multiple \\
\hline \multicolumn{7}{|l|}{ Address (Zoba) } \\
\hline & Maekel & 135 & 69 & 24 & 24 & 18 \\
\hline & Others & 17 & 6 & 1 & 5 & 5 \\
\hline & Missing & 4 & --- & 2 & --- & 2 \\
\hline \multicolumn{7}{|l|}{ Maternal age } \\
\hline & $<20$ & 15 & 10 & 1 & 4 & 0 \\
\hline & $20-24$ & 34 & 15 & 4 & 7 & 8 \\
\hline & $25-29$ & 59 & 29 & 8 & 12 & 10 \\
\hline & $30-34$ & 27 & 14 & 8 & 3 & 2 \\
\hline & $35-39$ & 15 & 6 & 5 & 1 & 3 \\
\hline & $40+$ & 4 & 0 & 1 & 2 & 1 \\
\hline & Missing & 2 & 1 & 0 & 0 & 1 \\
\hline \multicolumn{7}{|l|}{ Marital status } \\
\hline & Married & 73 & 34 & 13 & 12 & 11 \\
\hline & Single & 5 & 4 & 1 & 0 & 3 \\
\hline & Missing & 78 & 37 & 13 & 17 & 11 \\
\hline \multicolumn{7}{|l|}{ Ethnicity } \\
\hline & Tigrigna & 151 & 74 & 26 & 28 & 23 \\
\hline & Others & 4 & 0 & 1 & 1 & 2 \\
\hline & Missing & 1 & --- & --- & --- & --- \\
\hline \multicolumn{7}{|l|}{ Religion } \\
\hline & Christian & 73 & 34 & 14 & 12 & 13 \\
\hline & Muslim & 5 & 1 & 0 & 1 & 3 \\
\hline & Missing & 78 & 40 & 13 & 16 & 9 \\
\hline \multicolumn{7}{|l|}{ Parity } \\
\hline & 0 & 48 & 31 & 4 & 8 & 5 \\
\hline & 1 & 26 & 14 & 4 & 7 & 1 \\
\hline & 2 & 32 & 12 & 7 & 5 & 8 \\
\hline & 3 & 19 & 10 & 4 & 3 & 2 \\
\hline & $4+$ & 31 & 8 & 8 & 6 & 9 \\
\hline \multicolumn{7}{|l|}{ History of abortion } \\
\hline & 0 & 127 & 62 & 24 & 24 & 17 \\
\hline & 1 & 23 & 11 & 2 & 4 & 6 \\
\hline & 2 & 5 & 1 & 1 & 1 & 2 \\
\hline & $3+$ & 1 & 1 & 0 & 0 & 0 \\
\hline \multicolumn{7}{|l|}{ Year of Delivery } \\
\hline & 2007 & $8296 / 23$ & 9 & 6 & 7 & 1 \\
\hline & 2008 & $7744 / 26$ & 11 & 3 & 6 & 6 \\
\hline & 2009 & $8622 / 32$ & 15 & 3 & 8 & 6 \\
\hline & 2010 & $9433 / 42$ & 22 & 7 & 5 & 8 \\
\hline & 2011 & $5708 / 33$ & 18 & 8 & 3 & 4 \\
\hline \multicolumn{7}{|l|}{ Mode of delivery } \\
\hline & Vaginal & 134 & 75 & 25 & 16 & 18 \\
\hline & $\mathrm{C} / \mathrm{S}$ & 22 & 0 & 2 & 13 & 7 \\
\hline \multicolumn{7}{|l|}{ Sex of neonate } \\
\hline & Male & 62 & 19 & 13 & 16 & 14 \\
\hline & Female & 91 & 55 & 14 & 12 & 10 \\
\hline & Missing & 3 & 1 & --- & 1 & 1 \\
\hline \multicolumn{7}{|l|}{ Infant Weight (g) } \\
\hline & $<1000$ & 48 & 41 & 1 & 0 & 6 \\
\hline & $1000-2000$ & 37 & 25 & 5 & 2 & 5 \\
\hline & $2001-3000$ & 38 & 8 & 15 & 11 & 4 \\
\hline & $3001-4000$ & 23 & 1 & 5 & 12 & 5 \\
\hline & $4001+$ & 7 & 0 & 1 & 3 & 3 \\
\hline & Missing & 3 & --- & --- & 1 & 2 \\
\hline \multicolumn{7}{|l|}{ APGAR Score } \\
\hline & 0 & 116 & 75 & 11 & 13 & 17 \\
\hline & $1-6$ & 7 & 0 & 3 & 2 & 2 \\
\hline & $7+$ & 30 & 0 & 13 & 13 & 4 \\
\hline & Missing & 3 & --- & --- & 1 & 2 \\
\hline & Total & 156 & & & & \\
\hline
\end{tabular}


Cross tabulation was done to evaluate the association of NTD with different variables. Out of the variables of interest data on Marital status, ethnicity, religion, and maternal history of abortion were found to have no enough evidence for making conclusion. Because majority of the mothers who delivered in the hospital were from the Capital city and surrounding villages that stake the same socio - demographic and geographic area which is very near to the hospital.

Therefore, from the results of the study it is very difficult to make an association between address and NTD types. The results indicated that Majority 108 (69.2\%) of women who delivered neonates with NTD were below 30 years of age. The results also showed that most neonates with NTD were delivered from women who are less than Para four 125 (80.1\%). Mode of delivery was different for different types of NTD. All anencephalic were delivered vaginally while most hydrocephalic cases were delivered by cesarean section (44.8\%). Cesarean section rate for multiple abnormalities was higher (28\%) and spina bifida (7\%). Majority anencephalic neonates $66(88 \%)$ were born with weight of less than 2000 grams while significant neonates with hydrocephalus had weight greater than 2000 grams 26 $(92.9 \%)$. Majority of the neonates with Spina bifida had similar weight as normal neonates and multiple NTD neonates had a range of an overall distribution of birth weights. The results of APGAR score were founded to be zero for all anencephalic $75(0 \%)$ and majority $17(74 \%)$ of multiple NTD; while 40 percent of and 46 percent of neonates with spina bifida and hydrocephalus, respectively, had no APGAR at five minutes. Female sex was predominant among anencephalic neonates 55 (74.3\%) but both sexes had similar proportions for the other NTD types without any significant predominance. History of abortion or still birth had no association with NTD.

Over the years of the study an increase in the trend of anencephaly was observed which is statistically significant, whereas the other types of NTDs were in an increasing and decreasing pattern. There is a statistically significant increase in trend of total NTDs (anencephaly, spinabifida, hydrocephalus, and multiple anomalies) whereby for each month increase from 2007 to 2011, the increase was 1.5 percent $(p<0.05)$. The results of Poisson analysis indicated an exceptional statistically significant increase of anencephaly only, whereas for the other NTDs no any statistical significance was observed (Figure 1).

There was a statistically significant increase in trend of anencephaly whereby for each month increase from 2007 to 2011 , the increase is $2.0 \%$. $(p<0.05)$. Poisson analysis done for other types of NTD didn't show increasing trend over time (Figure 2). There was a statistically significant increase in trend of anencephaly whereby for each month increase from 2007 to 2011, the increase is $2.0 \%$. $(\mathrm{p}<0.05)$. Poisson analysis done for other types of NTD didn't show increasing trend over time (Figure 2).

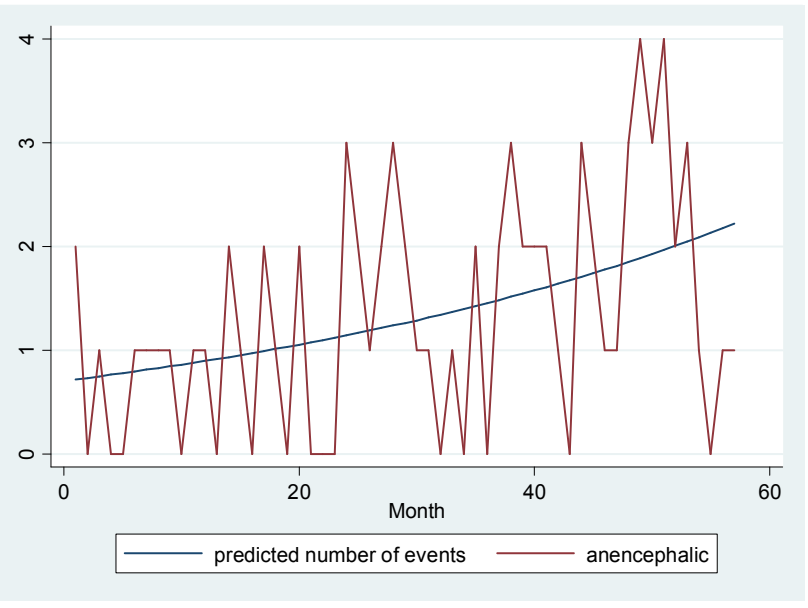

Figure 1. Poisson regression model of anencephaly over 56 Months.

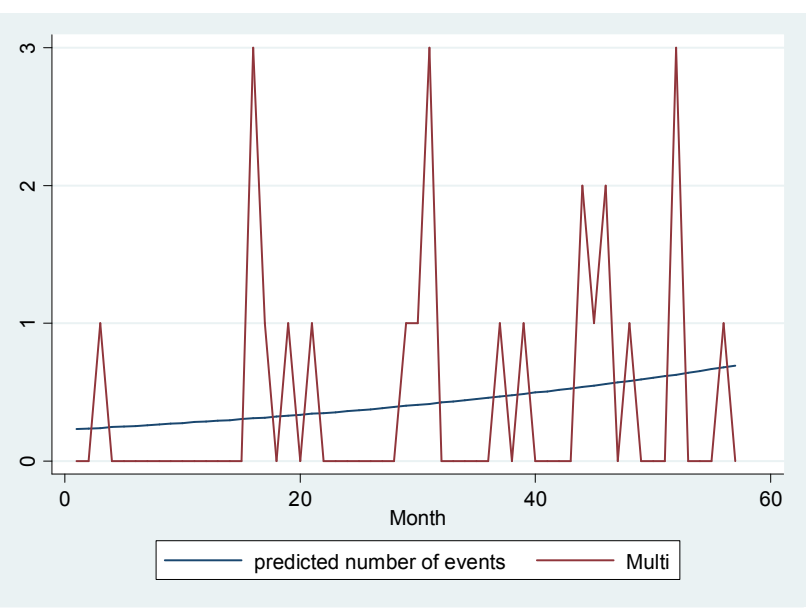

Figure 2. Poisson regression of combined abnormality with months.

Correlation was observed between the total number of deliveries and the increasing trend of NTD. Even though NTD deliveries were increasing with time there was no statistical significance of an increase in total deliveries over the 56 months of study period (Figure 3).

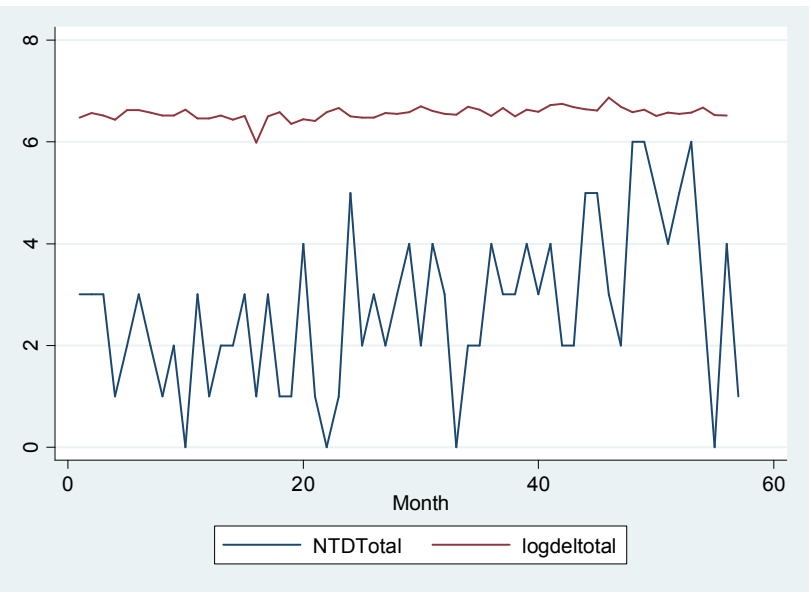

Figure 3. Graph total deliveries and NTD over time. 


\section{Discussion}

In this retrospective study the prevalence of NTD was relatively high as compared to most of the developed countries, which was around 1 per 1000. A study that was conducted in Europe by Khoshnood (2015), documented a total prevalence of 0.91 per 10000 for 1991-2011 and 0.85 per 10000 in the period 2003-07 which is very low as compared to the results of this study[7]. This high prevalence can be argued that as in Eritrea, even though there is folic acid supplementation, it is only during pregnancy as well as absence of folic acid fortification on foods can lead to this high prevalence of NTD [8]. This high result is similar to most developing world, where folic acid supplementation and prenatal counseling was not practiced $[9$, 10]. The result of the study showed anencephaly as the most common NTD. This is similar to most studies that showed indicated the same result. The increasing trend of NTD, specifically anencephaly is difficult to explain because it can be caused and it can be related to multifactorial reasons. The result of the study revealed that the higher anencephaly prevalence among female newborns which is consistent with other similar studies [11]. A study that was conducted in Khartoum, Sudan (2015), for assessing the prevalence of neural tube defects documented a prevalence of 2.8 per 1000; being higher in females (54.4\%) than males (45.6\%) [12].

It is not possible to explain the association of NTD with many of the variables including gestational age, marital status, ANC follow up, religion, and maternal history, because enough data were not extracted from records due to missing datas. Higher rates of NTD were documented as other similar studies in the in the age group of 25 to 35 . The relation with parity was found to be similar with those studies that had parity of less than four, but a study that was conducted in Texas documented a higher prevalence of NTD in those with a parity of less than two [13, 14]. Whether this is confounded with younger age is not clear. Similar finding was also seen between another study and our study without a relationship with those mothers with history of abortion and still birth. As hydrocephalus is clearly an indication for cesarean delivery it is not surprising that results of the study show higher rate of cesarean section with hydrocephalus and multiple abnormalities as hydrocephalus was one of the defects. The relationship of increased birth weight because of the bigger head mass is shown by a larger weight than other defects. The study also confirms anencephaly is incompatible with life but other newborns born with other defects may survive even later with further neurosurgical management. Major strength of the study is in the fact that is the first study of NTD and it was done not only for almost five years but in a busy hospital with more than 8000 delivery a year which is $50 \%$ of total delivery in the country.

In conclusion, the trend of NTDs in neonates was observed to increase significantly, mainly anencephaly. The study findings also noted that younger women (less than 30 years) and with parity of less than four were at higher risk as compared to those with an age above thirty and with a parity more than four.
Therefore, this retrospective record review study results highlight the need for a further prospective study and surveillance of NTD in the country. Because NTDs are diseases of public health importance which can be prevented and reduced with folic acid supplementation and fortification of principal foods that are consumed through the country.

\section{References}

[1] ACOG. Neural Tube Defects; ACOG Practice Bulletin, Clinical Management Guidelines, number 44, 2000.

[2] World Health Organization. Prevention of neural tube defects, Integrated Management of Pregnancy and Childbirth (IMPAC), Department of making pregnancy safer 2006.

[3] Pilu, G. \& Gabrielli, S., ClinicalObstetrics, $3^{\text {rd }}$ edition. Prenatal diagnosis of central nervous system malformations, Pages 347-358

[4] Adeleye, AO. et al. Central Nervous System Congenital Malformations in a Developing Country: Issues and Challenges against their prevention. Childs Nerv Syst 26:919924, 2010.

[5] Yin, Zhihua et al. A Population-based Case-Control study of risk factors for Neural Tube Defects in Shenyang, China. Childs Nerv Syst. 2010.

[6] Djientcheu, VP et. al. Management of Neural Tube Defects in a Sub-Saharan Country: The Situation in Yaounde, Cameroon. Journal of Neurological Sciences 275:29-32, 2008.

[7] Khoshnood B, Loane M, De Walle H, Arriola L, Addor MC, Barisic I, Beres J, Bianchi F, Dias C, Draper E, Garne E. Long term trends in prevalence of neural tube defects in Europe: population based study. Bmj. 2015 Nov 24; 351: h5949.

[8] Bestwick JP, Huttly WJ, Morris JK, Wald NJ. Prevention of neural tube defects: a cross-sectional study of the uptake of folic acid supplementation in nearly half a million women. PloS one. 2014 Feb 19; 9(2): e89354.

[9] Sayed, Abdul-Rauf et al. Decline in the Prevalence of Neural Tube Defects Following Folic Acid Fortification and Its CostBenefit in South Africa. Birth Defects Research (Part A) 82:211-216, 2008.

[10] Zaganjor I, Sekkarie A, Tsang BL, Williams J, Razzaghi H, Mulinare J, Sniezek JE, Cannon MJ, Rosenthal J. Describing the prevalence of neural tube defects worldwide: A systematic literature review. PloS one. 2016 Apr 11; 11(4): e0151586.

[11] Greene ND, Copp AJ. Neural tube defects. Annual review of neuroscience. $2014 \mathrm{Jul}$ 8; 37: 221-42.

[12] Omer IM, Abdullah OM, Mohammed IN, Abbasher LA. Prevalence of neural tube defects Khartoum, Sudan August 2014-July 2015. BMC research notes. 2016 Nov 24; 9(1):495.

[13] Mark A. Canfield et al. The prevalence and predictors of anencephaly and spina bifida in Texas. Peadiatric and perinatal epidemiology; 23: 41-50, 2009.

[14] Ahmed Z. Obiedat Neural tube defects in the Jordan: Is there a seasonal variation? Journal of child neurology; 25(7) 864-866, 2010 . 